\title{
UTILIZAÇÃO DE INSTRUMENTOS DO SISTEMA DE VIGILÂNCIA ALIMENTAR E NUTRICIONAL NA POPULAÇÃO IDOSA: REVISÃO BIBLIOGRÁFICA
}

Laura Virgili Claro, Universidade Federal de Santa Maria (UFSM), lauravirgili05@gmail.com Karla de Souza Maldonado da Silva, Universidade Federal de Santa Maria (UFSM), karllamaldonado@gmail.com

Raiane Dalmolin, Universidade Federal de Santa Maria (UFSM), raianedalmolin@yahoo.com Bruna Steffler, Universidade Federal de Santa Maria (UFSM), brunasteffler50@gmail.com Greisse Viero da Silva Leal, Universidade Federal de Santa Maria (UFSM), greisse@hotmail.com Vanessa Ramos Kirsten, Universidade Federal de Santa Maria (UFSM), kirsten.vr@gmail.com

\section{RESUMO}

Introdução: A Vigilância Alimentar e Nutricional é uma das diretrizes da Política Nacional de Alimentação e Nutrição, que identifica o consumo alimentar e estado nutricional da população, essas informações ainda são escassas em idosos. As políticas para o público materno-infantil majoritariamente foram responsáveis pelo maior registro de consumo alimentar no Sistema de Vigilância Alimentar e Nutricional (SISVAN). Objetivo: Realizar uma revisão bibliográfica sobre a utilização dos instrumentos (marcadores de consumo alimentar e estado nutricional) do SISVAN na população idosa. Métodos: Foram consultadas as bases de dados da Bireme e SciELO, utilizando os unitermos "consumo alimentar" AND "estado nutricional" AND "vigilância alimentar e nutricional". Foi adotado como critério de inclusão produções nos idiomas português e inglês, que contemplassem a utilização de dados do SISVAN e exclusão de revisões, teses, dissertações, documentos oficiais e duplicados. Resultados: Foram identificadas 11 publicações. Os instrumentos do SISVAN foram utilizados em 6 estudos com crianças e escolares (54,54\%), em 3 (27,27\%) com idosos, $1(9,09 \%)$ com mulheres beneficiárias do Bolsa Família e 1 (9,09\%) analisou dados nacionais. As publicações foram entre 2008 e 2020 , sendo a região sul com maior percentual $(54,54 \%)$ de utilização desses instrumentos. Dos estudos com os idosos, um não incluiu os marcadores, enquanto que as pesquisas com os outros públicos estudados todos utilizaram. Conclusão: No Brasil, ainda não há uma política de alimentação e nutrição direcionada aos idosos, no entanto o monitoramento das condições de saúde desse público é um dispositivo importante para o desempenho de intervenções e consolidação do SISVAN.

Palavras-chave: Envelhecimento; vigilância nutricional; sistemas de informação; saúde pública. 